\title{
Viabilidad del desarrollo de una Comunidad de Seguridad desde el Consejo de Defensa Suramericano
}

\author{
Viability of the development of a Security Community from the South \\ American Defence Council
}

\author{
Sebastián Koch Merino* \\ Centro de Estudios Estratégicos de la Academia de Guerra (CEEAG) Santiago, Chile
}

Recibido: 22 de diciembre de 2015. Aprobado: 9 de mayo de 2016.

\begin{abstract}
Resumen
Utilizando el Análisis de Política Exterior (APE), coadyuvado por el constructivismo como teoría de las Relaciones Internacionales se estudiará, en el marco de la Unión de Naciones Suramericanas (Unasur), la viabilidad del establecimiento de una Comunidad de Seguridad desde el Consejo de Defensa Suramericano (CDS). Además de los elementos racionalistas de la definición de política exterior de los Estados, se emplearán las perspectivas reflexivas de las Relaciones Internacionales, ya que son parte esencial del proceso de establecimiento de una Comunidad de Seguridad. Las identidades, valores y significados compartidos, adquieren un valor tan importante como los objetivos racionales, siendo la relación entre ambos elementos la que permitiría, bajo ciertas circunstancias, el establecimiento de una Comunidad de Seguridad en la región.
\end{abstract}

Palabras claves: Comunidad de Seguridad, Unión de Naciones Suramericanas, constructivismo.

Cientista Político, Universidad Diego Portales; estudiante del programa de Doctorado en Ciencia Política, Universidad Diego Portales; diplomado en Estudios Europeos, Universidad de Concepción; diplomado en Seguridad Humana, Universidad de Łódź, Polonia. Investigador y analista del Centro de Estudios Estratégicos de la Academia de Guerra (CEEAG). Correo electrónico: sebastian.koch@mail.udp.cl 


\begin{abstract}
Using Foreign Policy Analysis (FPA), helped by constructivism as an international relations theory, this paper studies the establishment of a Security Community by the South American Defence Council (SDC), within the Union of South American Nations (USAN). Besides the rationalist elements involved in the definition of States' foreign policy, reflexive perspectives from international relations will be employed, since they are an essential part of the process for establishing a Security Community. Shared identities, values and meanings, become as important as rational objectives, being the relationship between these two elements what would allow, under certain circumstances, the establishment of a Security Community for the region.
\end{abstract}

Keywords: Security Community, Union of South American Nations, constructivism.

\title{
Introducción
}

La interacción estatal en Latinoamérica, especialmente la cooperación, ha sido fomentada por los Estados de la región desde hace ya varias décadas. Existen alrededor de 17 organismos regionales/subregionales en América Latina -algunos datan incluso desde fines de la década de 1960 y principios de los 70-, que varían en su composición y en los objetivos que persiguen; entre otros, objetivos políticos, económicos, comerciales. Pese a la abundancia de organismos, pareciera que la región no es capaz de llegar a un nivel de cooperación cercano al de la Unión Europea (UE).

En el plano de la Seguridad y la Defensa, un avanzado nivel de interacción y cooperación estatal derivaría en el establecimiento de una Comunidad de Seguridad (Deutsch, 1957, p. 124; Adler y Barnett, 1998, pp. 3-4; Flemes, Nolte y Wehner, 2011, p. 107).

La Organización del Tratado del Atlántico Norte (OTAN) podría ser considerada como el gran ejemplo a este respecto. La OTAN, subsistente hasta nuestros días, ha sido capaz de lograr una institucionalidad de Seguridad y Defensa en común para sus miembros, contemplando procedimientos para la solución pacífica de controversias -entre ellosy una defensa colectiva en caso de amenazas y ataques externos. La misma lógica es seguida por el Tratado Interamericano de Asistencia Recíproca (TIAR) de 1947. Estas dos 
instituciones no sólo nacieron con anterioridad al concepto de Comunidad de Seguridad, sino que además siguen una lógica distinta. Se condicen con el concepto de seguridad colectiva, concentrando sus esfuerzos en medidas de defensa colectiva ante amenazas o ataques externos a la región correspondiente, mientras que en el caso de una Comunidad de Seguridad se está ante un caso de seguridad cooperativa. En el caso del TIAR, se habla de un ejemplo de seguridad hemisférica colectiva (De la Lama, 1998, pp. 45-46), caracterizado por la consideración de una agresión a un Estado de la región como una agresión a todos los Estados que la componen y por una respuesta colectiva a dicha agresión, en virtud del derecho de legítima defensa de la carta de la Organización de Naciones Unidas (ONU) (De la Lama, 1998, p. 46). En cambio, una Comunidad de Seguridad es más cercana a la visión de seguridad hemisférica cooperativa. Se relaciona con evitar el surgimiento de nuevos conflictos a través del fomento de medidas que efectivas o no- propicien la confianza entre los Estados de la región (De la Lama, 1998, pp. 52-53). A través de la interacción de las distintas políticas gubernamentales, se busca la prevención y contención de las distintas amenazas a dichos Estados y a sus intereses nacionales, evitando así que la percepción que los actores tienen de éstas se traduzca en tensión, crisis o confrontación con otros miembros de la región (Varas, citado en De la Lama, 1998, pp. 53-54).

Se entiende entonces que la seguridad colectiva -teniendo una íntima relación con la lógica imperante durante la Guerra Fría- es reactiva en cuanto a las amenazas y ataques externos y contempla medidas de respuesta solidarias y colectivas para sus miembros, mientras que la seguridad cooperativa sigue una lógica de prevención de conflictos que se centra en el desarrollo institucional previo al surgimiento de una amenaza a los intereses nacionales racionales de los Estados correspondientes. Por lo mismo, un proceso de cooperación en temas de Seguridad y Defensa que apunte a la generación de una institucionalidad para evitar el surgimiento de conflictos entre sus miembros a partir de las amenazas externas -seguridad cooperativa- está íntimamente relacionado con el proceso de generación de una Comunidad de Seguridad. Mientras más desarrollado se encuentre este proceso de cooperación, más cerca debiera estar una región determinada de establecer una Comunidad de Seguridad (al menos en el plano teórico).

Teniendo esto en mente y pensando en la región sudamericana, es posible plantear la siguiente interrogante: ¿es viable el establecimiento de una Comunidad de Seguridad a partir del Consejo de Seguridad (CDS) de la Unión de Naciones Suramericanas (Unasur)? Esto se analizará y se explicará desde los elementos teóricos de las Comunidades de Seguridad y desde el Análisis de Política Exterior (APE), complementado con los elementos de la teoría constructivista (lógica planteada por Álvarez, 2015, p. 47). De 
esta manera se incorporan al análisis los elementos racionales tradicionales propios del APE y los elementos reflexivos de las ideas y de los entendimientos en común, propios del constructivismo. Finalmente, se concluye que si bien se han hecho esfuerzos para avanzar en el camino del establecimiento de una Comunidad de Seguridad, dicho avance ha sido bajo, por lo que la región se encuentra aún muy lejos del establecimiento de una Comunidad de Seguridad propiamente tal desde el CDS de Unasur.

\section{Análisis de política exterior}

El APE analiza la política exterior de los Estados con miras a determinar cuáles -y en qué medida- son los elementos que influyen o al menos afectan en la determinación de ésta (Álvarez, 2015, p. 49). Se dedica además a predecir la conducta de las colectividades humanas que componen el Estado, desde la perspectiva de una teoría decisional humana en política (Hudson y Vore, 1995, p. 209). Para comprender cómo son tomadas las decisiones en política exterior, parte de la base que los individuos, sea de manera aislada o agrupada, son el origen de gran parte de las conductas y cambios en relaciones internacionales (Hudson y Vore, 1995, p. 209).

La política exterior de un Estado puede entenderse como un programa -guiado por objetivos o por problemas- confeccionado y seguido por las autoridades respectivas, dirigido a entidades externas a éste (Hermann, 1990, p. 5). La política exterior no es constante, puede sufrir cambios cuando menos, en cuatro niveles: (1) cambios por ajustes, (2) cambios de programa, (3) cambios de problema/objetivo, (4) cambios en la orientación internacional (Hermann, 1990, p. 5). El grado en el que cambia la política exterior de un Estado depende de tres dimensiones: (1) el grado de institucionalización, o el compromiso del gobierno con su política exterior, (2) el grado de apoyo u oposición de los actores internos a la política exterior, y (3) el grado de prominencia o de significancia del tema en cuestión en el juego de poder interno (Hermann, 1990, p. 7). El comprender que la política exterior no es fija permite, por ejemplo, que esté sujeta a corrección en caso de que su desempeño no sea el esperado: si en un área determinada de la política exterior se evidencia una baja efectividad, es posible escanear dicho problema, identificar la causa de la inefectividad y llevar a cabo medidas correctivas (Hermann, 1990, p. 9).

El APE desmiente la idea -desorientadora, para Putnam (1988, p. 433) - de que los Estados son actores unitarios, reconociendo que existen distintos niveles de agregación en cuanto a la composición estatal. El plano interno del Estado se desagrega en los 
distintos grupos/entidades/actores, con potestades en lo que respecta a las relaciones internacionales (Hudson y Vore, 1995, p. 210). Por lo mismo, se vuelve necesario entender las particularidades de los individuos involucrados en las decisiones de política exterior, así como de los grupos reducidos que participan de las decisiones de política exterior y de los procesos organizacionales y burocráticos involucrados en el proceso de toma de decisiones de política exterior (Hudson y Vore, 1995, p. 216). En el APE se rompe con la visión clásica del Estado como un actor unitario, como un todo indivisible y se reconoce no sólo la existencia, sino que la pertinencia de las distintas unidades de agregación en la definición de la política exterior.

El APE considera también el factor externo al Estado. Putnam (1988, p. 434), al referirse a los procesos de la política internacional, establece que pueden ser entendidos como un juego de dos niveles: un nivel nacional, compuesto por grupos domésticos y por políticos con sus respectivos intereses, y un nivel internacional, compuesto por los diversos actores internacionales. Esto vuelve compleja la definición de la política exterior, debiendo buscar una consistencia entre el nivel interno y el externo, aun cuando a veces los intereses que priman en uno pueden ser contrarios a los del otro (Putnam, 1988, p. 434). Gourevitch (1978) llega al punto de establecer que el sistema internacional no sólo es una consecuencia de la política doméstica, sino que es a su vez una causa de ésta; existe tanto una codependencia como una interrelación fuerte entre ambos elementos, por lo que deben, para él, ser analizados como un todo (Gourevitch, 1978, p. 911). Putnam se refiere además al win-set, que estaría compuesto por los distintos resultados en el nivel externo que serían aceptados en el nivel interno; un win-set más amplio haría más fácil un acuerdo en el nivel externo (Putnam, 1988, p. 437).

Moravcsik (1997, p. 513) analiza la influencia de las ideas en el comportamiento de los Estados, estableciendo que a través de éstas son moldeadas las preferencias estatales; las ideas, vistas como propósitos sociales fundamentales, son parte importante del cálculo estratégico llevado a cabo por los gobiernos. A través de estos cálculos estratégicos, los Estados definirán sus preferencias y las plasmarán en su política exterior, asunto que para el autor es de primera importancia en las relaciones internacionales (Moravcsik, 1997, p. 513).

Entonces, el constructivismo reconoce que el Estado es un actor compuesto por una agregación de niveles y elementos que se relacionan entre sí. Los distintos elementos que lo componen -desde el individuo, pasando por las instituciones, hasta quien toma las decisiones en política exterior- están marcados por una serie de ideas y elementos cognitivos que determinan sus intereses racionales materiales, intereses que muchas 
veces no coinciden incluso en un mismo Estado, complejizando así el establecimiento de su política exterior. Si a esto se agrega la influencia del factor externo en la determinación de la política exterior, ésta verdaderamente se convierte en un difícil juego de dos niveles, en el que debe buscarse no sólo mantener un equilibrio entre el nivel interno y el externo, sino que además buscar un win-set amplio y aprovecharlo para así tener un mayor margen de acción en la definición de la política exterior. Lo recién dicho y la valoración del plano de las ideas en la política exterior, va a permitir la incorporación del constructivismo al APE.

\section{Constructivismo}

Las teorías reflexivas de las Relaciones Internacionales se centran en el análisis del plano de las ideas y en cómo la convergencia/divergencia en torno a éstas afecta los intereses y el comportamiento de los Estados en la política internacional (Álvarez, 2015, p. 51). Dentro de estas teorías se encuentra el constructivismo. Éste se ocupa de la construcción social de la política mundial (Wendt, 1995, p. 71). Los entendimientos intersubjetivos son concebidos como un conocimiento colectivo, compartido por quienes son competentes en un ámbito determinado (Cohen citado en Adler, 1997, p. 327) y no como una mera agregación de posturas individuales. Ve las estructuras de la política mundial como sociales, admitiendo que además del comportamiento, tanto la identidad como los intereses de los actores son moldeados por las estructuras internacionales (Wendt, 1995, pp. 71-72).

Los Estados se desenvuelven, tanto en un ambiente social como en uno material, con actores internacionales que influyen en la constitución de la estructura internacional y viceversa (Álvarez, 2015 p. 56). Para Hopf (1998, p. 172), el programa de investigación del constructivismo se enfoca en las identidades existentes en la política mundial, tanto a nivel internacional como a nivel interno -dentro de los Estados-, y en cómo éstas van a influir en el comportamiento de los Estados como actores internacionales.

Para el constructivismo existe una correlación -bidireccional- entre los actores y la estructura internacional: ésta influye en el comportamiento de los actores y éstos, a través de sus acciones y de sus prácticas, no únicamente se reproducen a sí mismos en la estructura en cuestión, sino que reproducen (o no) a la estructura misma (Hopf, 1998, p. 172; Adler, 1997, p. 330). Esta constitución mutua se basa en una serie de elementos del plano de las ideas -entendimientos, expectativas, conocimientos- 
compartidos por los Estados. Los recursos materiales que conforman una estructura únicamente cobran importancia a través del valor social que le dan los actores (Wendt, 1995, p. 73). Los elementos tradicionalmente reconocidos en la estructura internacional van a ser valorados y, por lo mismo, determinados en su importancia a través de las ideas y del entendimiento que los Estados tengan de éstos; no tienen un valor indiscutible en sí mismos, sino que son las estructuras cognitivas las que le dan significancia e importancia al mundo material (Adler, 1997, pp. 319 y 322). Desde el minuto en que un elemento material sea visto como poco importante, éste pierde su valor, el que podría recuperarse a través de apreciaciones futuras que los actores hagan de él.

La combinación de estos elementos debe traducirse en procesos e instituciones y no quedarse en lo netamente discursivo; debe darse un moldeamiento de la estructura internacional, de sus procesos de tomas de decisiones y de los espacios en que se comparten y moldean dichos entendimientos. Wendt (1995, p. 74) llega al punto de decir que desde el momento en que se deje de actuar según una idea, la estructura que surgía de dicha idea se acaba. Ante una eventual inexistencia de ideas articuladoras en la estructura internacional, el mundo estaría sumergido en una incertidumbre peor que la provocada por la anarquía internacional (Hopf, 1998, pp. 174-175). En cambio, al existir ideas compartidas y significados intersubjetivos, las prácticas sociales generadas a partir de éstas producen y reproducen las estructuras, reduciendo la incertidumbre internacional (Hopf, 1998, p. 178). La cooperación internacional es posible aun estando en un escenario internacional anárquico, en torno a áreas determinadas distribuidas según las identidades y los intereses de los Estados. A medida que los Estados van cooperando, las comunidades intersubjetivas se van reconstruyendo, retroalimentando y fortaleciendo (Hopf, 1998, pp. 189-190).

En base a lo recién analizado, se entiende que los elementos intersubjetivos influyen en la generación de la política exterior de los Estados, en especial en cuanto a cooperación internacional. La política exterior va a depender de la existencia de precedentes intersubjetivos y de material simbólico compartido en el momento de interpretar eventos, de silenciar otras alternativas de interpretación de esos mismos eventos, de establecer las prácticas de la estructura y de orquestar el accionar colectivo (Ashley citado en Hopf, 1998, p. 179). Ésta es la idea que permite aplicar el planteamiento de Álvarez (2015) en cuanto a la utilización del constructivismo para complementar el APE. 


\section{Constructivismo y análisis de política exterior}

Debido a que el constructivismo toma en consideración tanto los elementos racionales como los reflexivos, permite comprender las distintas motivaciones del accionar estatal en cuanto a política exterior, permitiendo comprender las variaciones en los niveles de entendimientos compartidos entre los miembros de una estructura internacional (Álvarez, 2015, p. 57).

Por su parte, el APE considera cinco aspectos psicológicos: (1) características individuales, (2) percepciones, (3) sociedad y cultura, (4) el sistema político, y (5) el sistema internacional (Hudson y Vore, 1995, p. 226). El segundo aspecto psicológico -el más relacionado con el constructivismo- comenzó a ser considerado como influyente en la política exterior de los Estados desde los fines de la Guerra Fría (Hudson y Vore, 1995, p. 226). Esta estrecha relación con los postulados del constructivismo permite fundamentar la complementación del APE con este paradigma. A través de la consideración de lo racional y de lo reflexivo, el constructivismo complementa y enriquece el APE al asumir los elementos racionales propios de éste, pero problematizándolos en base a elementos propios del plano de las ideas y de las identidades (Álvarez, 2015, pp. 58-59).

La interrogante planteada por Gonzalo Álvarez permite fundamentar esta complementación: “¿Cómo explicar que estados con intereses convergentes -que puedan beneficiarse mutuamente de una relación cooperativa- tiendan al conflicto?" (Álvarez, 2015, p. 51).¿Por quéexisten Estados que tienen intereses -racionales-afines, pero que pese a eso adolecen de una ausencia de cooperación? Aquí entra en juego el constructivismo: a través de la expansión del corolario de fenómenos estudiados, es posible determinar las causas de la inexistencia de cooperación en sectores donde evidentemente sería más provechoso cooperar. Los intereses racionales de los Estados parecieran ser incapaces de explicar todas las decisiones tomadas por éstos como actores de las relaciones internacionales, dando cabida a la capacidad explicativa del constructivismo en cuanto a los componentes inmateriales de la política exterior.

Las ideas influyen en los distintos ámbitos de la estructura doméstica (Gourevitch, 1978, pp. 882-883); los elementos constructivistas parecieran influir en las distintas funciones internas de los Estados, como por ejemplo, en la definición de su política exterior. Los entendimientos e ideas compartidos -tanto a nivel interno como a nivel externo-influyen en las decisiones tomadas por los Estados. A nivel externo, pareciera que los Estados con elementos reflexivos afines se agrupan y cooperan entre sí, excluyendo a los que no comparten sus lineamientos. A nivel interno, el componente humano del Estado ejerce 
presiones en base a sus planteamientos inmateriales. Los Estados deben aprovechar los entendimientos compartidos que existen y priman en su interior, para generar políticas afines a éstos y buscar que sus decisiones en política exterior sean aceptadas por el nivel interno: deben aprovechar el win-set de su componente reflexivo.

Hasta ahora se buscó aclarar la perspectiva desde la que se analizará la eventualidad del surgimiento de una Comunidad de Seguridad en la región. La combinación del APE y del constructivismo resulta útil para analizar un sinfín de fenómenos del escenario internacional. En el caso de este artículo en particular, la viabilidad del establecimiento de una Comunidad de Seguridad en la región.

\section{Comunidad de seguridad}

El concepto de Comunidad de Seguridad surge del trabajo de Karl Deutsch (1957). Es una comunidad política que a través de la cooperación busca eliminar la expectativa de guerra dentro de sus límites, generando una sensación de comunidad e instituciones que aseguren las expectativas de cambio pacífico -solución de problemas a través de procedimientos institucionalizados- por un largo período de tiempo. Existe -en la teoríauna certeza de que sus miembros no van a enfrentarse entre sí a través de la fuerza física para dirimir sus controversias, sino que recurrirán a otras alternativas (Deutsch, 1957, pp. 123-124). Además, existe un conocimiento compartido por parte de los miembros conformado por una identidad e ideas en común (Wendt citado en Álvarez, 2015, p. 58), manifestando en sus elementos fundacionales una clara tendencia constructivista.

El concepto es profundizado por Adler y Barnett (1998) quienes, reconociendo las observaciones de Deutsch, establecen que en las Comunidades de Seguridad existe una sensación de comunidad -determinada por valores compartidos que son retroalimentados a su vez por estas comunidades- que asegura que sus miembros resolverán sus diferencias de maneras distintas a la guerra (Adler y Barnett, 1998, p. 4). Una Comunidad de Seguridad debe contemplar tanto una renuncia a la violencia militar entre sus miembros como hábitos fuertemente enraizados de solución pacífica de controversias (Adler y Barnett, 1998, p. 35).

Existen tres capas -dentro de las que se avanza gradualmente- en el proceso de formación y desarrollo de una Comunidad de Seguridad: (1) condiciones que precipiten una 
interacción entre Estados, (2) factores que conduzcan al desarrollo de confianza mutua y de una identidad colectiva, y (3) condiciones necesarias para expectativas confiables de cambio pacífico, de confianza mutua y de identidad común (Adler y Barnett, 1998, p. 38). En la segunda capa, tanto el poder como el conocimiento tienen una gran importancia como elementos que propician la cooperación y eventualmente el surgimiento de una Comunidad de Seguridad, al permitir el desarrollo de visiones colectivas y de la generación de identidades en común (Adler y Barnett, 1998, pp. 39-45). A partir de estos elementos se puede generar la confianza necesaria -esencial para asegurar las expectativas de cambio pacífico (Adler y Barnett, 1998, pp. 46-48).

Las Comunidades de Seguridad pasan por tres fases en su proceso de desarrollo y establecimiento: (1) la fase naciente, (2) la fase ascendente y (3) la fase madura (Adler y Barnett, 1998, pp. 49-58). En la primera fase -naciente- se da un primer acercamiento entre Estados, en cuanto a la evaluación de alternativas de cooperación para mejorar sus condiciones en el escenario internacional, en específico, en el ámbito de Seguridad y Defensa.

La segunda fase -ascendente- está caracterizada por un aumento en la densidad de las redes de cooperación entre los miembros: instituciones, organismos y organizaciones. Además, la percepción de los demás Estados miembros como una amenaza disminuye drásticamente (Adler y Barnett, 1998, p. 53). Comienzan a surgir estructuras cognitivas en común, en especial en cuanto a la percepción y manejo de las amenazas, estrechando los lazos militares (Adler y Barnett, 1998, p. 55). Se profundizan y potencian los acercamientos iniciados a partir de la primera fase y se traducen en instancias de cooperación concretas, tanto en el plano institucional como en el operacional.

La tercera y última fase -madura- se da cuando los miembros de la Comunidad de Seguridad tienen una identidad común, con expectativas confiables de cambio pacífico. Aquí es cuando la Comunidad de Seguridad comienza a existir como tal. Dentro de la misma se dan distintos patrones de conducta que la retroalimentan y fortalecen. Se potencia también la solución pacífica a eventuales controversias, dándose un mayor desarrollo institucional en este ámbito. La guerra -sin dejar de existir como posibilidades cada vez una alternativa menos plausible entre sus miembros (Adler y Barnett, 1998, p. 55).

Las Comunidades de Seguridad son un ejemplo de cooperación internacional en temas de Seguridad y Defensa. La eliminación de la guerra como una alternativa para la solución de controversias dentro de los límites de ésta, es una situación que claramente 
reporta un beneficio para todos sus miembros. Entonces, recordando la pregunta planteada por Álvarez (2015, p. 51), ¿por qué si los beneficios racionales de establecer una Comunidad de Seguridad resultan tan evidentes no ha sido posible establecerla en la región? ¿Son suficientes los aspectos netamente racionales? ¿Qué se puede decir acerca del eventual establecimiento de una Comunidad de Seguridad en Sudamérica? Pareciera que los requisitos teóricos de éstas van más allá de los intereses y de las capacidades de los Estados de la región. Éstos son excedidos a tal punto, que ni siquiera se da un desarrollo de la identidad en común, condición necesaria para empujar todo este proceso.

\section{CDS y Unasur bajo esta visión}

Desde el término de la Guerra Fría, los países de Sudamérica han apuntado a la cooperación en varios ámbitos, incluido el de Defensa. A través de interacción internacional reiterada se busca potenciar la solución pacífica de conflictos en la región, apuntando a la construcción de un régimen regional de seguridad (Briones, 2013, p. 2). Se busca generar una instancia regional de cooperación en temas de Seguridad y Defensa que disminuya la incertidumbre, las posibilidades de guerra y que fomente la solución pacífica de controversias en Sudamérica.

Dicho objetivo se mostró imposible, especialmente durante la Guerra Fría, debido a la alta injerencia de Estados Unidos (EEUU) en la región; los intereses estadounidenses se manifestaron en instituciones como la Organización de Estados Americanos (OEA) y se entiende que las definiciones de los Estados latinoamericanos en términos de Seguridad y Defensa estaban fuertemente influidas por los intereses de Estados Unidos. Terminada la Guerra Fría, surgieron en la región una serie de valores, conceptos, ideas e ideales que no se condicen en su totalidad con los valores estadounidenses, debido a un cambio en el foco de la política exterior norteamericana (Briones, 2013, pp. 3-4). Esto no quiere decir que debido a la presencia de intereses estadounidenses no se haya dado una búsqueda de la paz, sino que busca retratar el surgimiento de una identidad propiamente sudamericana que no necesariamente va de la mano con los ideales de EEUU.

A partir de estos ideales se constituye Unasur, como una institución que -en teoríamaterializa la voluntad de cooperación sudamericana. Por lo mismo, busca una mayor cooperación en temas culturales, sociales, económicos y políticos a nivel sudamericano. Se muestra como la plataforma ideal para la confluencia en cuanto a los elementos reflexivos, potenciando -o buscando potenciar- la influencia del plano de las ideas en la 
definición de la política exterior de los Estados de la región. Dentro de Unasur, el CDS se constituye como una entidad para la promoción, en el escenario internacional, de una voz común regional en temas de Seguridad y Defensa (Briones, 2013, pp. 12-13). Esto pareciera quedarse en el plano teórico. En muchas ocasiones, los miembros de Unasur son guiados por sus intereses materiales unilaterales en desmedro de los vestigios de una identidad compartida. Esto se da especialmente en materias de Seguridad y de Defensa, plano en el que ciertos casos de cooperación bilateral no se han traducido en intentos multilaterales de cooperación. Esto derivó en que dentro de la región exista una serie de agendas nacionales individuales, separadas unas de otras, que apuntan a intereses -materiales y reflexivos- divergentes entre los Estados de Sudamérica, adoptando una lógica de interés nacional en lugar de una lógica de interés regional (Flemes, Nolte y Wehner, 2011, p. 114).

La inclusión de la identidad sudamericana está relacionada con los elementos reflexivos rescatados por el constructivismo, pero pareciera que las identidades y valores en Sudamérica no son compartidos por todos. Existen vestigios de una identidad sudamericana, pero pese a ello cada Estado tiene una fuerte tendencia a seguir sus propios intereses nacionales de manera unilateral, en desmedro de los intentos de cooperación internacional. Por lo mismo, hay quienes plantean que pese a los esfuerzos que se han realizado desde el CDS de Unasur para establecer una Comunidad de Seguridad, la región aún está lejos de lograr dicho cometido, principalmente debido a la existencia de intereses materiales e ideológicos divergentes, lo que dificulta la profundización de los esfuerzos de cooperación en los planos de Seguridad y Defensa (Flemes et al., 2011, pp. 107-108). Es de igual manera posible sostener que, en vista de los elementos constructivistas en el APE y de los requisitos esenciales para el establecimiento de una Comunidad de Seguridad, el CDS de Unasur ha avanzado - poco, pero ha avanzado- en el establecimiento de los primeros vestigios de una identidad sudamericana que vaya más allá de un inocuo discurso político.

Para referirse a los aspectos de carácter práctico del CDS, es necesario remitirse a su estatuto. En él se indica que el CDS se constituye como un órgano "de consulta, cooperación y coordinación en materia de Defensa” (Unasur, 2008). Además de suscribirse a los principios establecidos en la carta de la ONU, en la de la OEA y a mandatos y decisiones tomados en Unasur, el CDS declara como suya una serie de principios expuestos a lo largo de su artículo 3 (Unasur, 2008). Para los motivos de este artículo es relevante recordar los principios consagrados en las letras c) -promoción de la paz y de la solución pacífica de controversias-, d) -fortalecimiento del diálogo en materias de defensa a través de las medidas de confianza mutuas y de la transparencia-, i) -fomento a la reducción 
de las asimetrías en materias de defensa en la región en orden a fortalecer la capacidad de la región en defensa-, 1) -promoción de iniciativas de cooperación en defensa reconociendo las distintas realidades de los Estados de la región-, y m) -convivencia pacífica de los pueblos y otros elementos derivados de ésta-, relacionados directamente con las funciones propias de una Comunidad de Seguridad; y el plasmado en la letra j) -fomento a la defensa soberana de los recursos naturales de las naciones-, vinculado con los planteamientos constructivistas al reconocer, a través de la diferenciación respecto de los actores externos, a las naciones de la región.

Asimismo, se vuelve necesario considerar los objetivos de la organización, explicitados en el artículo 4 del estatuto; las tres letras de éste se relacionan tanto con los elementos teóricos de una Comunidad de Seguridad como con los postulados constructivistas: a) -consolidación de Sudamérica como zona de paz-, b) -construcción de una identidad suramericana en materias de defensa-, c) generación de consensos para potenciar la cooperación en defensa en la región.

Además de la evidencia teórica encontrada en el estatuto, es provechoso poner atención al Protocolo de Paz, Seguridad y Cooperación del CDS de Unasur. Al ya mencionado organismo se le encargó, en el año 2009, la tarea de generar un protocolo que buscara una profundización del proceso de integración en la región, especialmente en materias de Seguridad y Defensa, poniendo énfasis en la promoción de medidas de confianza mutua, en la generación de políticas de transparencia en materias de Seguridad y Defensa, en el rechazo de la amenaza y del uso de la fuerza, en el respeto de la integridad territorial y la soberanía de los miembros de Unasur, en la no intervención en los asuntos internos y en la solución pacífica de controversias (Inostroza y Pezzano, 2014, p. 1). La delegación de Perú hizo una presentación sobre este tema en la XXII reunión del Consejo de Delegados, el 25 de noviembre del año 2009 (Inostroza y Pezzano, 2014, p. 7). Dicha presentación tuvo como antecedentes las ideas debatidas en una reunión extraordinaria de jefas y jefes de Estado en Bariloche, Argentina, un par de meses antes. Además, la ya mencionada presentación iba de la mano con las ideas planteadas en un documento presentado por la delegación de Chile, que apuntaba a la generación de una arquitectura de seguridad para Unasur (Inostroza y Pezzano, 2014, p. 7). Para enero de 2010, Perú se habría ofrecido como líder de las negociaciones en este ámbito, lo que fue aprobado por todas las demás delegaciones, creándose el grupo de trabajo para la elaboración de dicho protocolo (Inostroza y Pezzano, 2014, p. 7).

Las reuniones del grupo de trabajo (cuatro, hasta el año 2014) estuvieron marcadas por la discusión de diversos aspectos del protocolo: su naturaleza jurídica, estructura 
capitular y contenido de los mismos, entre otros (Inostroza y Pezzano, 2014, p. 8). Al tratar tres temas en particular puestos en la agenda a pedido de la delegación venezolana -naturaleza jurídica, definición del concepto de seguridad y marco institucional dentro del que se insertaría el protocolo-, comenzaron a evidenciarse posturas distintas entre algunas delegaciones (Inostroza y Pezzano, 2014, pp. 8-12), lo que permite plantear que, efectivamente, los intereses propios de cada uno de los Estados miembros parecieran pesar más que la búsqueda de un consenso regional, y de ideas e identidades compartidas en torno a estos temas. Así, impulsados por sus realidades nacionales, cada país propuso, en torno a diversos temas, planteamientos que volvían difícil una coordinación hacia ideas en común. Todo esto hizo que se llegara a un punto en que aún no está terminado el protocolo en cuestión, lo que ha puesto un freno al proceso de integración en materias de Seguridad y Defensa. Y, como ya se dijo, es necesario que las iniciativas de integración pasen más allá de su formulación en el plano reflexivo; es necesario que éstas se traduzcan en iniciativas concretas (Wendt, 1995, p. 74), tales como instituciones, normas y otros elementos que trascienden desde lo reflexivo hacia lo material.

Por ello, es posible estimar que el cumplimiento de uno de los objetivos del CDS, la generación de una identidad de seguridad compartida por los miembros de Unasur, aún se encuentra distante. Retomando la pregunta de Álvarez (2015), los Estados que evidentemente se verían beneficiados por una cooperación en temas en que existen intereses convergentes, a veces no cooperan debido a que se hace un foco excesivo en los elementos racionales de la fallida cooperación y no se toman en consideración las identidades compartidas y los elementos cognitivos en común de los Estados en cuestión. La cooperación sería posible -en la teoría- a través de la inclusión de un fuerte arraigo de ideas en común (Flemes et al., 2011, p. 113). El problema está en esa idea: además de que no existe dicho arraigo en Sudamérica, en las pocas oportunidades en que se manifiestan vestigios de ese arraigo, los intereses racionales materiales de los Estados priman por sobre el elemento reflexivo, tomando decisiones unilaterales que apuntan a la maximización de dichos intereses. Ejemplo de esto es lo planteado por Sanahuja (2011, pp. 142-143), quien establece que si bien es posible evidenciar consensos sólidos en Unasur en materias de regionalismo y de integración, en otros ámbitos esta solidez no se manifiesta, sea porque dos Estados que el autor considera hegemónicos en la región -Venezuela y Brasil- no convergen en sus intereses, o bien, porque los intereses que sí convergen corresponden a países menos influyentes que los dos recién mencionados, lo que deriva en una situación en la que es difícil promover la agenda integradora. De existir efectivamente una verdadera identidad sudamericana en materias de Seguridad y Defensa, bastaría con el reconocimiento de dichas identidades para que la agenda se 
impulsara por sí sola, dado que, como ya vimos, los elementos reflexivos inciden en los elementos racionales (Adler, 1997, pp. 319 y 322).

Hay quienes hablan de poder material, determinado por los beneficios y costos materiales, y de poder ideacional-normativo, marcado por las prácticas sociales que serán parte del proceso de cooperación internacional (Flemes et al., 2011, p. 114). Es necesario generar una correlación entre ambos poderes para siquiera soñar en superar la incongruencia entre la teoría y la realidad. Aun entonces, dada la tendencia en la región y el poco desarrollo de los elementos identitarios en común, es posible que los intereses relacionados con el poder material opaquen al poder ideacional-normativo -como lo ya mencionado en cuanto al grupo de trabajo para la confección del Protocolo de Paz, Cooperación y Seguridad-, reproduciendo el comportamiento estatal que apunta a la persecución de intereses racionales materiales de manera unilateral. Si se apunta a la creación eventual de una Comunidad de Seguridad, debe equilibrarse la valoración de los elementos identitarios y de los elementos materiales. Centrarse únicamente en el ámbito material volverá imposible el nacimiento de una Comunidad de Seguridad, debido a que no se tiene en consideración la identidad compartida que debe existir entre los miembros; centrarse únicamente en el ámbito de las ideas también trae un costo aparejado, ya que por muy similares que sean las identidades entre dos o más Estados, por muy compartido que esté el conocimiento, difícilmente entablarán algún proceso de cooperación efectivo ante la inexistencia de intereses materiales. La utilización del APE junto al constructivismo para analizar esta falencia permite tener un mayor margen de acción en el plano teórico, obteniendo mayor capacidad explicativa de los fenómenos, pero difícilmente puede dar una receta infalible para el establecimiento de una Comunidad de Seguridad en la región. Como se dijo, esto dependerá fundamentalmente -debido a que se está trabajando en el plano de Seguridad y Defensa- del comportamiento de los Estados en base a sus intereses materiales y los elementos reflexivos influirán únicamente hasta cierto punto. Los Estados, en el momento de definir su política exterior, no se concentran de manera única en los elementos materiales, sino que a éstos se agrega la consideración del plano valórico, pero pareciera que la explicación constructivista necesita de un mínimo de constitución identitaria en común para operar, situación que no se ha dado en Sudamérica.

$\mathrm{Al}$ analizar la constitución de identidades en común desde Unasur, ésta está relacionada con la búsqueda de elementos que cohesionen a los miembros de la eventual Comunidad de Seguridad (Flemes et al., 2011, p. 115). La existencia de dichos elementos es discutible debido al peso que tienen los intereses materiales unilaterales en la política exterior sudamericana, especialmente en el plano de Seguridad y Defensa. Dicha búsqueda se ha caracterizado más por excluir los intereses de actores externos a la región que por 
buscar cohesionar a los actores regionales. Se ha enfocado en un otherness u othering (Hansen, 2006, citado en Flemes et al., 2011, p. 115; Wehner, 2010, citado en Flemes et al., 2011, p. 115), proceso marcado por una fuerte distinción entre aquellos actores parte de Unasur y aquellos externos a ésta. A lo que se llegó es a una clara distinción entre los actores sudamericanos y los extra sudamericanos, pero no a una identidad sudamericana en común. Si bien se hace una distinción hacia fuera, no ha propendido con igual éxito a la cohesión de identidades hacia dentro.

En lo estrictamente teórico pareciera que el caso de Unasur y de su CDS va de la mano con los elementos contemplados por el constructivismo, por el APE y en cuanto a las Comunidades de Seguridad, pero en la realidad práctica dicha situación no se aprecia. Los intereses discordantes existentes entre los miembros de Unasur evitan que el CDS sea capaz de generar una Comunidad de Seguridad, ya que cada Estado apunta hacia la arista de la agenda regional que más se condice con sus intereses racionales materiales, relegando los elementos reflexivos a un segundo plano. Pareciera que los intereses unilaterales de los Estados miembros tienen una mayor capacidad de determinación de su comportamiento que el plano de las ideas. De ser así, es necesario analizar si dicho fenómeno se produce debido a la inexistencia de una clara identidad sudamericana en común o debido a la preponderancia de lo material por sobre lo reflexivo, restándole validez a la percepción constructivista de que el valor del elemento material de las relaciones internacionales viene dado por el valor que dicho elemento tiene en el plano de las ideas para los actores internacionales y reforzando los preceptos racionales de la Teoría de las Relaciones Internacionales.

Ahora, pese a que la generación de una Comunidad de Seguridad aún no se ha logrado, es correspondiente reconocer que la Unasur, atendiendo a su papel como mecanismo de gestión de crisis (Sanahuja, 2011, p. 130), ya ha actuado en varias ocasiones. Como destacan Inostroza y Pezzano (2014), Unasur ha sido parte en el tratamiento de una serie de controversias -Bolivia en 2008, Honduras en 2009, la controversia por las bases estadounidenses en Colombia en 2009, Venezuela y Colombia desde 2010, Ecuador en 2010, Paraguay en 2012, y a lo largo del proceso de paz entre Colombia y las FARC (Inostroza y Pezzano, 2014, p. 5). Más allá de su mera participación, hay autores que estiman que, en otro momento, ${ }^{1}$ las ya mencionadas situaciones en las que participó Unasur habrían generado un quiebre institucional o incluso un conflicto militar, lo que permite destacar el importante rol de Unasur en lo que respecta a coordinación y

El empleo de la expresión "en otro momento" puede ser vinculado con la participación de Unasur, por lo que podría entenderse que las posibles consecuencias que los autores enuncian habrían sucedido de no ser por la participación de Unasur. 
búsqueda de una solución pacífica ante las controversias (Colombo y Roark, 2012, p. 34). Entonces, pese a que aún queda camino por recorrer en cuanto a lo relacionado con la construcción de una identidad sudamericana, es necesario reconocerle a Unasur el importante rol que ha jugado en el camino hacia uno de sus objetivos, relacionado directamente con las Comunidades de Seguridad: la consolidación de Sudamérica como una zona de paz.

\section{Conclusiones}

El establecimiento de una Comunidad de Seguridad en la región es una idea que ni siquiera puede considerarse como un objetivo. No es parte de la agenda de todos los miembros de Unasur. Si bien en el discurso político los Estados suelen hacer declaraciones grandilocuentes acerca de la identidad sudamericana y los sentimientos de hermandad existentes en ésta, estos discursos no se plasman -en los ámbitos de Seguridad y Defensa- en la realidad. Hoy en día no existe en la región una clara identidad sudamericana. Podría decirse que lo que existe es una identidad extrasudamericana y en base a esta diferenciación nos concebimos como miembros de la región. Pero no existe un entramado de ideas compartidas que cohesione la identidad sudamericana hacia el interior, dificultando enormemente la efectividad explicativa del constructivismo como un elemento determinante en la conformación de la política exterior de los Estados. Es más, pareciera que en la región los Estados siguen sus lineamientos racionales de manera unilateral la mayoría de las veces, siendo pocas las situaciones que están marcadas por la existencia de una identidad en común que guíe la política exterior de los Estados, su accionar y sus decisiones en el escenario internacional.

Hoy en día, no existe en la región una Comunidad de Seguridad y estamos aún lejos de tener una a nivel sudamericano. Pese a ello, se reconocen ciertos avances en cuanto a su establecimiento, los que son dignos de ser mencionados y analizados. Dichos avances dicen relación con la búsqueda de una identidad sudamericana en común -que se plasme en los ámbitos de Seguridad y Defensa-, con el hecho de que la Unasur ya haya intervenido en una serie de controversias en pos de la solución pacífica de las mismas, y principalmente con el proceso de institucionalización de prácticas conjuntas en materia de Seguridad y Defensa. A través del CDS es posible soñar con un eventual establecimiento de una Comunidad de Seguridad, a partir de la profundización de la cooperación en materias de Seguridad y Defensa, y del análisis en común de ciertos fenómenos a este respecto. Los Estados deben además poner atención al ya mencionado win-set, jugando con el 
establecimiento de ideas imperantes a nivel interno y posteriormente enmarcando su política exterior dentro de dichas ideas. Es necesario generar a nivel interno la idea de que el establecimiento de una Comunidad de Seguridad es necesario, debido a los riesgos que su inexistencia implica, y debido a los beneficios que su eventual establecimiento traería aparejados.

Éstas son algunas de las medidas que podrían propiciar en Sudamérica el establecimiento de una Comunidad de Seguridad propiamente tal, pero ello no quiere decir que sea una receta que asegure el éxito de este proceso. Son factores que podrían catalizarlo, coadyuvarlo, pero no por ello son condiciones suficientes para su establecimiento. En la realidad, resulta difícil establecer un catálogo de medidas que, fundamentadas en la teoría, conduzcan indiscutidamente a la creación de una Comunidad de Seguridad, aún más cuando varios de los elementos que dicha teoría contempla no se manifiestan por completo en la región.

Si retomamos los planteamientos de Adler y Barnett (1998, pp. 49-58), es posible declarar que la región sudamericana, en cuanto al establecimiento de una Comunidad de Seguridad, se encuentra en las etapas iniciales de la fase ascendente, recién terminando la fase naciente: ya se dieron los primeros acercamientos en búsqueda de la cooperación en materias de Seguridad y Defensa, así como se generaron las primeras instituciones y normas que han de regirla; pero la cooperación aún no es lo suficientemente densa y las estructuras cognitivas en común no están lo suficientemente generalizadas y afianzadas, lo que se traduce -por ejemplo- en procesos inconclusos de generación de normas e instituciones.

Es por ello que se considera que el establecimiento de una Comunidad de Seguridad propiamente tal- a partir del CDS de Unasur es viable, pero en un largo plazo, debiendo antes recorrer un camino marcado por avances en torno a los intereses materiales divergentes de los Estados y a avances en torno a una identidad sudamericana en común que llegue a los ámbito de Defensa y Seguridad. De darse avances en los dos planos recién mencionados, acompañados de procesos de institucionalización en los que estos elementos reflexivos se traduzcan en elementos y procesos materiales concretos -normas e instituciones, entre otros-, es posible que eventualmente llegue a constituirse una Comunidad de Seguridad en nuestra región. 


\section{Referencias}

Adler, E. (1997). Seizing the middle ground: Constructivism in world politics. European Journal of International Relations 3 (3), 319-363.

Adler, E. y Barnett, M. (1998). Introduction and theoretical overview. En E. Adler y M. Barnett (eds.), Security Communities (pp. 1-65). Cambridge: Cambridge University Press.

Álvarez, G. (2015). Hacia una perspectiva constructivista y crítica del análisis de política exterior. Estudios Internacionales 47 (180), 47-65.

Briones, D. (2013). América Latina: Desafíos para la integración en Seguridad y Defensa. Documento de Opinión 53, 1-14. Disponible en http://www.ieee.es/Galerias/fichero/ docs_opinion/2013/DIEEEO53-2013_DesafioSeguridad_DanielaBriones.pdf

Colombo, S. y Roark, M. (2012). Unasur: integración regional y gobernabilidad en el siglo XXI. Densidades 10, 21-40.

Deutsch, K. (1957). Political community and the North Atlantic Area. En N. Brent y A. Stubb (eds.), The European Union (pp. 121-143). Londres, Inglaterra: Lynne Rienner Publishers.

De la Lama, J. (1998). La seguridad hemisférica a fin de siglo: el nuevo concepto de seguridad hemisférica cooperativa. Santiago, Chile: FLACSO-Chile.

Flemes, D., Nolte, D. y Wehner, L. (2011). Una comunidad de seguridad regional en formación: la Unasur y su Consejo de Defensa. Estudios Internacionales 44 (170), 105-127.

Gourevitch, P. (1978). The second image reversed: The international sources of domestic politics. International Organization 32 (4), 881-912.

Hermann, C. (1990). Changing course: When governments choose to redirect foreign policy. International Studies Quarterly 34 (1), 3-21.

Hopf, T. (1998). The promise of constructivism in international relations theory. International Security 23 (1), 171-200.

Hudson, V. y Vore, C. (1995). Foreign policy analysis yesterday, today and tomorrow. Mershon International Studies Review 39 (2), 209-238. 
Inostroza, M. V. y Pezzano, L. (2014). El Proyecto de Protocolo de Paz, Seguridad y Cooperación de la Unasur: perspectivas y posibilidades. Presentado en el VII Congreso de Relaciones Internacionales del Instituto de Relaciones Internacionales de la Universidad Nacional de La Plata.

Moravcsik, A. (1997). Taking preferences seriously: A liberal theory of international politics. International Organization 51 (4), 513-553.

Putnam, R. (1988). Diplomacy and domestic politics: The logic of two-level games. International Organization 42 (2), 428-460.

Sanahuja, J. A. (2011). Multilateralismo y regionalismo en clave suramericana: El caso de Unasur. Pensamiento Propio 33 (1), 115-158.

Unasur (2008). Estatuto del Consejo de Defensa Suramericano de la Unasur. Recuperado http://www.unasursg.org/images/descargas/ESTATUTOS\%20CONSEJOS\%20 MINISTERIALES\%20SECTORIALES/ESTATUTO\%20CONSEJO\%20DE\%20 DEFENSA.pdf

Wendt, A. (1995). Constructing international politics. International Security 20 (1), 71-81. 